\title{
Detection of Lipomannan in Cattle Infected with Bovine Tuberculosis
}

\author{
Dung M. Vu,* Rama M. Sakamuri,*W. Ray Waters, ** Basil I. Swanson, ${ }^{*}$ and \\ Harshini MUKUNDAN*广 \\ *Chemistry Division, MS J567, Los Alamos National Laboratory, Los Alamos, NM 87545, USA \\ **National Animal Disease Center, Agriculture Research Service, US Department of Agriculture, Ames, \\ IA 50010, USA
}

\begin{abstract}
Early and rapid detection of bovine tuberculosis (bTB) is critical to controlling the spread of this disease in cattle and other animals. In this study, we demonstrate the development of an immunoassay for the direct detection of the bovine bTB biomarker, lipomannan (LM) in serum using a waveguide-based optical biosensor. We apply an ultra-sensitive detection strategy developed by our team, termed lipoprotein capture, that exploits the pull-down of high-density lipoprotein (HDL) nanodiscs from cattle blood that allows for the recovery and detection of associated LM. We also profile the change in the expression of these TB biomarkers as a function of time from a small set of samples collected from studies of bovine TB-infected cattle. We demonstrate for the first time the direct detection of bovine LM in serum, and clearly show that the biomarker is expressed in detectable concentrations during the entire course of the infection.
\end{abstract}

Keywords Lipomannan, lipoarabinomannan, lipoprotein capture, waveguide-based biosensor

(Received February 14, 2017; Accepted February 25, 2017; Published April 10, 2017)

\section{Introduction}

Bovine tuberculosis (bTB) is a chronic infectious disease caused by Mycobacterium bovis. While bTB primarily affects cattle and other animals, humans can also be susceptible to $M$. bovis. Currently, the standard control measure applied to bTB is testing and slaughter. ${ }^{1,2}$ The Caudal Fold Tuberculin test (CFT) is one of the primary technologies used by the US dairy farmers and cattle ranchers to screen their herds. ${ }^{1,2}$ This test requires skin injection of a small amount of tuberculin, a purified protein derivative from $M$. bovis, followed by visual interpretation of the skin after $72 \mathrm{~h}$. Early testing of M. bovis infection, desensitization, exposure to environmental mycobacteria species, and other factors can contribute to the ambiguous read-out of the tuberculin skin test in cattle. ${ }^{1-3}$ In addition to this, visual inspection in darker-colored animals can sometimes be challenging. ${ }^{2}$ Confirmation of the tests via laboratory culture can be time-consuming (requiring 4 to 6 weeks), ${ }^{1,4}$ which can leave farmers and ranchers unsure of what to do with animals yielding inconclusive test results. Also, culturing of $M$. bovis is challenging and not always successful. These limitations, can result in either infected animals being undetected, or the unnecessary quarantine and slaughtering of uninfected ones. Additional tests have been introduced, but none of them have demonstrated the requisite simplicity, sensitivity and specificity to overcome the current limitations. . $^{2,5} 6$ Therefore, rapid and sensitive blood-based detection methods for the early detection of bTB are clearly needed, and could eliminate stress to

$\dagger$ To whom correspondence should be addressed.

E-mail: harshini@lanl.gov livestock, reduce economic burden and pave the way forward for the elimination of bTB in the national cattle herd of the United States and elsewhere.

We have developed a waveguide-based optical biosensor for the rapid, sensitive and specific detection of pathogenic biomarkers, and have effectively employed it for the detection of biomarkers associated with numerous diseases. ${ }^{7-11}$ This biosensor relies on evanescent sensing on a planar waveguide surface to amplify the transduction signal of the fluorescently labeled antibodies to the target antigen.7,12 We have also developed novel surface functionalization chemistries and target pull-down strategies to increase the sensitivity and specificity of our assays. ${ }^{10,13-16}$ Specifically, our work has targeted the detection of pathogen-specific biomarkers with exquisite sensitivity and specificity, with minimal sample processing, so as to facilitate the rapid point-of-care diagnosis of infection. In this study, we adapted assays originally developed for the human TB lipoarabinomannan (LAM) biomarker to its bovine counterpart, lipomannan (LM), on the wave-guide based biosensor platform.

Several pathogen-specific biomarkers for bTB have been identified. Noteworthy among them are LM and early secretory antigenic target 6, ESAT6. ${ }^{1,17-20}$ LM is an abundant lipoglycan component of the $M$. bovis cell envelope, and is secreted by the metabolically active bacterium during infection, which results in activation of the host immune response. ${ }^{1,17,18}$ The amphiphile is well-conserved in evolution and like its counterpart secreted by M. tuberculosis, LAM, LM is known to be an immune activator. ${ }^{1,17,18}$ In our ongoing work with developing diagnostics for human TB, we discovered that LAM could be readily detected in urine, ${ }^{9}$ whereas its direct measurement from blood was more elusive. ${ }^{14}$ Work from our laboratory revealed that this 
difficulty is associated with the biochemistry of LAM in serum, for the amphiphile is unstable in monomeric form in aqueous blood, and is consequently sequestered by carrier molecules, like high-density lipoproteins (HDL). ${ }^{14}$ This observation resulted in the development of our lipoprotein capture assay for the pull-down of HDL nanodiscs using antibodies that target apolipoprotein A1 (ApoA1), the coat protein of HDL. Subsequent detection of LAM associated with HDL using the anti-LAM antibody in blood resulted in a sensitive and direct strategy for measurement of this signature. ${ }^{14}$ Herein, we have successfully transitioned our HDL capture assay from human TB LAM to bTB LM. We demonstrated the application and sensitivity of our biosensor platform to the detection of LM in blood from a small set of samples collected from studies of bTB infected cattle. Our work demonstrates the first step towards developing reliable point-of-care molecular diagnostics for the rapid diagnosis and prognosis of bTB. Thus, this is by no means a comprehensive and statistically viable demonstration of a diagnostic assay, but merely a validation of the feasibility of the lipoprotein capture methodology for the detection of amphiphilic LM in infected cattle.

\section{Experimental}

\section{Reagents and samples}

A cocktail of three anti-LAM monoclonal antibodies (clones 24,27 , and 171) was used as reporter antibodies, which was a generous gift from the Foundation for Innovative New Diagnostics (FIND, Geneva, Switzerland). Additional information about these antibodies can be obtained upon request by the FIND. Purified LM was procured from a materials contract from the Colorado State University Materials Consortium (via BEI Resources). LEZ-link sulfo-NHS-LC-LCbiotin and NeutrAvidin were purchased from Thermo Scientific. Alexa Fluor 647 (AF647) labeling kit was obtained from Invitrogen (Thermo Fisher Scientific). Anti-apolipoprotein A1 antibody (anti-ApoA1) and biotinylated anti-ApoA1 antibody were purchased from AbCam. 1,2-Dioleoyl-sn-glycero-3phosphocholine (DOPC) and 1,2-dioleoyl-sn-glycero-3phosphoethanolamine-N-(cap-biotinyl) (cap-biotin-PE) were from Avanti Polar Lipids, Inc. Miniature G-25 sephadex columns were acquired from Harvard Apparatus and bovine serum was purchased from Hyclone Laboratories. All other assay reagents were from Sigma-Aldrich, unless otherwise specified.

\section{Waveguide-based optical biosensor}

The waveguide-based sensor was developed at the Los Alamos National Laboratory and is described in detail elsewhere. ${ }^{7}$ The planar silicon oxynitride ( $\mathrm{SiONx}$ ) optical waveguides are fabricated at nGimat Ltd (Atlanta) and are coated with a 10-nm surface of $\mathrm{SiO}_{2}$ to enable functionalization at Spectrum Thin Films Inc. Details of these waveguides are described elsewhere. ${ }^{12}$

\section{Preparation of labeled antibodies}

The individual FIND antibodies and neutravidin were labeled with an Alexa Fluor 647 (AF647) dye using a fluorescence labeling kit from Invitrogen, as per the manufacturer's instructions. The labeled antibody was separated from unlabeled antibody by gel filtration, and tested for activity using an immunoblot assay. The concentration of the labeled antibody and the degree of labeling were measured by ultra-violet visible (UV-Vis) spectroscopy. The capture antibody, anti-ApoA1 (100 $\mathrm{nM}$ ) was purchased in biotinylated form and used as is.

\section{Cleaning, assembling and functionalization of waveguides}

Waveguides and glass coverslips were cleaned by sequential sonication in chloroform, ethanol, and water, followed by exposure to UV-light and ozone (40 min). After cleaning, a double-sided adhesive silicon gasket with a laser cut channel was used to assemble the waveguide to the glass coverslip. Following assembly, the flow cell was injected with a preparation of lipid micelles (prepared as described below). The functionalized waveguide flow cells were stabilized overnight before use.

The lipid micelles were prepared as described before ${ }^{10,16}$ from a thin-film preparation of $5 \mathrm{mM}$ DOPC and 1\% cap-biotinyl-PE (in chloroform); the chloroform was evaporated under argon. The lipid thin-film was then rehydrated in phosphate buffered saline solution (PBS) buffer for $60 \mathrm{~min}$. The sample was then subjected to 8 freeze-thaw cycles with liquid nitrogen and warm water and sonicated with a probe tip sonicator for $5 \mathrm{~min}$ to form uniform vesicles.

\section{Detection of LM by lipoprotein capture immunoassay}

The lipoprotein capture assay utilized an antibody directed against the coat protein of ApoA1 as the capture antibody. This allows for the capture of LM associated with HDL nanoparticles in serum, as described earlier for other targets. ${ }^{14}$ A subsequent detection using a cocktail of fluorescently labeled antibodies (anti-FIND-LAM-AF647, clones 24, 27 and 171) allows for the detection of LM associated with HDL nanoparticles in serum. These antibodies were specifically chosen from a selection of $>5$ clones because of their ability to bind conserved regions of lipomannan, and thereby recognize the target with excellent sensitivity. Development of targeted antibodies for LM, which discriminate it from LAM, will be required to improve the sensitivity of the diagnostics in the future.

In all experiments, waveguides functionalized with lipid bilayers were mounted on the flow cell, and blocked with a $2 \%$ bovine serum albumin (BSA)-PBS solution for $60 \mathrm{~min}$. The waveguide-associated background (intrinsic measure of impurities associated with the instrument) was then measured. All waveguide experiments were performed at room temperature (RT). After each addition, the flow cell was washed with $0.5 \%$ BSA in PBS. Then, fluorescently labeled neutravidin (NeuAF647, $100 \mathrm{pM}$ ) was injected into the flow cell (5 min), washed, and fluorescence intensity was measured to confirm the integrity of the biotinylated lipid bilayer surface, and served as an intrinsic positive control for system configurations in each experiment. Then, unlabeled neutravidin $(10 \mathrm{nM})$ was added to saturate the functional surface.

The incubation times for the antigens and antibodies were optimized in all cases by standard measurements using LM spiked into commercially procured bovine serum. The reporter antibodies were first evaluated for their ability to bind LM using the standard immunoblot methodology, which has been described before.9,16 Antigen and antibody titrations were performed on the waveguide-based sensor. LM was spiked into commercial serum for $24 \mathrm{~h}$, to allow for complete association with serum lipoproteins, and the signal intensity was measured with the antibodies at various times. Based on this, the normalized experimental protocol for clinical measurements was decided as such: the biotinylated anti-ApoA1 antibody (100 nM, $10 \mathrm{~min}$ incubation) was added as the capture antibody. Non-specfic (NS) interactions were determined by the addition of the reporter antibody cocktail (clones 24, 27 and 171 antiFIND-AF647, $20 \mathrm{nM}$ each, $15 \mathrm{~min}$ incubation). Then, the bovine serum sample was added to the flow cell and the sample was incubated for $60 \mathrm{~min}$. The reporter antibody cocktail was 


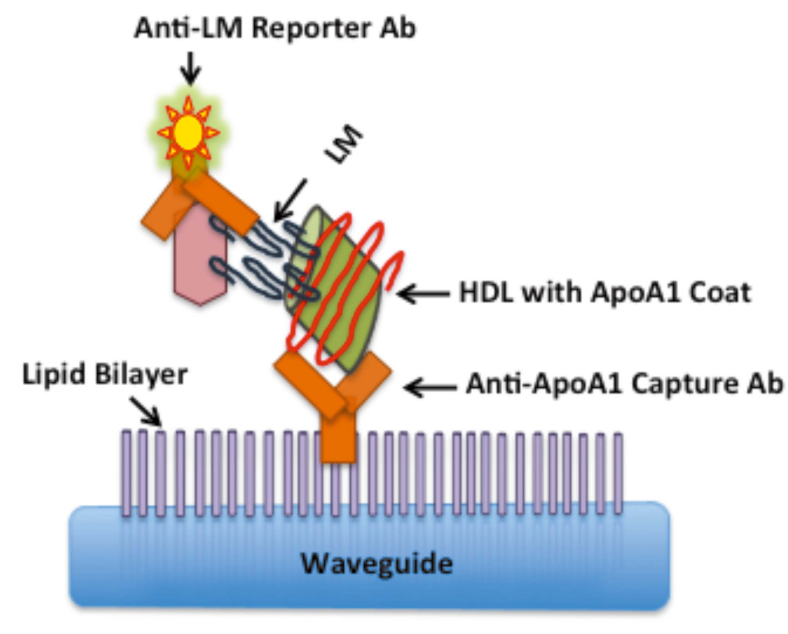

Fig. 1 Schematic representation of the lipoprotein capture assay.

re-added to the flow cell $(15 \mathrm{~min})$, this time to measure the specific fluorescence signal associated with binding of the LM biomarker to the reporter using a spectrometer interface.

\section{Evaluation of clinical samples}

The cattle samples were obtained from Dr. Waters at the US Department of Agriculture (USDA). The 7, 14, 21 days and 5-month cattle blood samples were obtained from the following studies. The 7-day samples came from a 2012 study of 23 cattle to compare differential pathology for different $M$. bovis strains. The 14-day samples came from 2007 study of 32 cattle to monitor the vaccination efficacy between an attenuated $M$. bovis Ravenal strain compared to the Bacillus Calmette-Guerin (BCG) vaccine from a Danish strain. The 21-day samples came from a 2010 study of 15 cattle to study vaccination efficacy. The 5-month samples came from a 2006 study of 5 cattle with the subjects challenged with $M$. bovis. For each of these studies pre-challenge samples and post-challenge samples were collected and stored at minus $80^{\circ} \mathrm{C}$. Unless noted, at least two pre-challenge and post-challenge cattle blood samples were tested from each of these studies at the various infection time points. The results were plotted for each animal individually, to accommodate the animal-to-animal variability. Thus, the difference of the post-challenge minus pre-challenge values for each animal, at the time point under considerations was taken as a measure of positivity or negativity.

\section{Results and Discussion}

Measurement of serum LM by lipoprotein capture assay

We evaluated the detection of LM in cattle blood samples at varying time points after the steers were challenged with M. bovis. Figure 1 shows a schematic representation of detection of LM by lipoprotein capture assay on the waveguide based optical biosensor. Figure 2 shows the detection of LM in blood in a steer before being challenged with M. bovis and 14 days post-challenge. Again the associated background and the NS specific binding signal associated with the interaction of the antibody with the surface are minimal $(<400 \mathrm{rfu})$, relative to both the pre- and post-challenge LM associated fluorescence signal. We also evaluated infected cattle samples at 1 week, 3 weeks, and 5 months for detection of the LM biomarker in these samples (Table 1, Fig. 3). In all of these samples, we were able

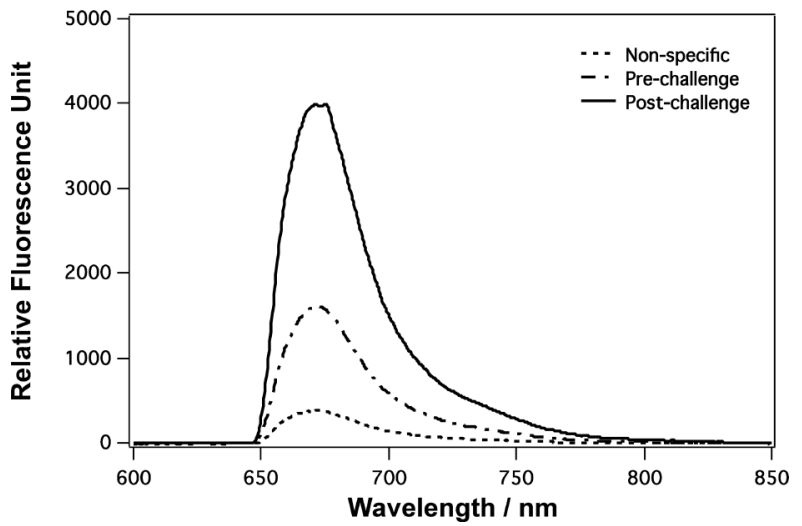

Fig. 2 Detection of LM in blood in a steer sample before and 14 days after infection.

Table 1 Summary of LM detection by HDL capture assay

\begin{tabular}{lcccc}
$\begin{array}{c}\text { Time after } \\
\text { infection }\end{array}$ & $\begin{array}{c}\text { Pre-challenge/ } \\
\text { rfu }\end{array}$ & $\begin{array}{c}\text { Post-challenge/ } \\
\text { rfu }\end{array}$ & $\begin{array}{c}\text { Difference/ } \\
\text { rfu }\end{array}$ & $\begin{array}{c}\text { Standard } \\
\text { deviation }\end{array}$ \\
\hline 7 Days & 600 & 3200 & 2600 & a \\
14 Days & 1358 & 2950 & 1593 & 714 \\
21 Days & 1563 & 2853 & 1290 & 1117 \\
5 Months & 975 & 1520 & 545 & 361 \\
\hline
\end{tabular}

a. Denotes measurement derived from one dataset.

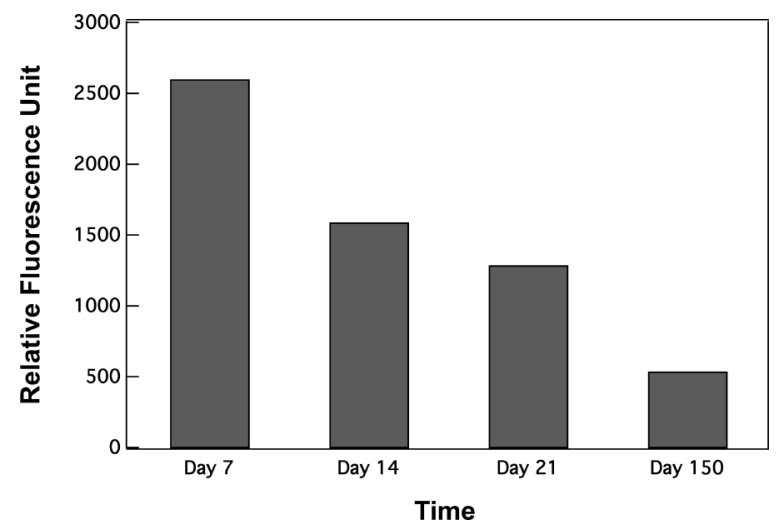

Fig. 3 LM biomarker profile of cattle with bTB.

to detect LM in the post-challenge animals. LM concentrations are highest 7 to 14 days after infection, and decrease at 21 days and 5 months post-challenge, as shown in Fig. 3.

LM is an amphiphilic lipoglycan, which is unstable in monomeric form in the aqueous milieu of the host. Consequently, several investigators have attempted to measure the carbohydrate components of this molecule in urine. ${ }^{21-24}$ This strategy has worked both in humans and animal, ${ }^{21-24}$ but suffers from some major limitations: 1) the sugar components of LM in urine may or may not be antigenic, because of their processing and delipidation during the renal clearance process, and 2) given the uncertain molecular weight of the components of LM in urine, it is not possible to quantify the observations. To this end, we determined that detection of LM in blood can overcome these challenges. This detection, however, is associated with several challenges, associated with the biochemistry of the molecule 
and its inherent instability in blood. Our team has developed two assay methods (membrane insertion and lipoprotein capture) and adapted them for the measurement of amphiphiles like LAM and lipopolysacharides. ${ }^{10,14,16}$ We have shown here that one of these methods can be applied to LM as well, provided that suitable antibodies are available.

There are several novel aspects of this work: 1) it demonstrates that bovine LM is also associated with lipoproteins in cattle sera, which establishes a similarity of host-pathogen interaction in this species, 2) our method shows that unlike host biomarker studies, pathogen biomarker-based measurements can be used in human and animal hosts, and thereby conforms to global One Health Strategy (https://www.cdc.gov/onehealth/) of diagnostics, and 3) we demonstrate for the first time the direct detection of bovine LM in serum, and clearly show that the biomarker is expressed in detectable concentrations during the entire course of the infection.

There are still many limitations to this work that need to be addressed in the future. For one, we performed this work with available banked pre- and post-challenge cattle sera from positive control infected animals of vaccination studies conducted at the USDA. A more systematic measurement of signals from the same animals, at different time points, in statistically viable numbers is required to establish the expression profile. The use of other biomarkers such as ESAT6 and antigen 85 complex will strengthen the outcomes of this effort. We are currently working on ESAT6 detection assays and measurement of ESAT6 antibodies for cattle.

\section{Conclusions}

We have demonstrated the ability to directly detect pathogen biomarkers associated with bovine tuberculosis from cattle infected with the disease very early after infection. We are confident the combination of the novel lipoprotein capture pull-down assays developed by our team, and the ultra-sensitive waveguide-based detection platform will therefore allow for a rapid and early diagnosis of infection from bovine tuberculosis.

\section{Acknowledgements}

This work was supported by funding from the New Mexico Small Business Assistance (NMSBA) Program, the USDA and the Los Alamos Research and Development (LDRD), Directed Research Program (Integrative Biosurveillance, Harshini Mukundan and Benjamin McMahon). The authors thank the FIND for the antibodies used in this research and the BEI Resources Materials Consortium for the Lipomannan antigen.

\section{References}

1. B. Admassu, E. Kebede, and A. Shite, Eur. J. Biol. Sci., 2015, 7, 169.

2. B. M. Buddle, G. W. de Lisle, W. R. Waters, and H. M. Vordermeier, in "Tuberculosis, Leprosy and Mycobacterial Diseases of Man and Animals", ed. H. Mukundan, M. A. Chambers, W. R. Waters, and M. H. Larsen, 2015, CAB International, Oxfordshire, UK, 168.
3. M. L. Monaghan, M. L. Doherty, J. D. Collins, J. F. Kazda, and P. J. Quinn, Vet. Microbiol., 1994, 40, 111.

4. D. J. V. Beste, M. Espasa, B. Bonde, A. M. Kjerzek, G. R. Stewart, and J. McFadden, PLOS ONE, 2009, 4, e5349.

5. D. F. Ramos, P. E. A. Silva, and O. A. Dellagostin, Braz. J. Biol., 2015, 75.

6. S. A. J. Strain, J. McNair, and S. W. J. McDowell, "Bovine Tuberculosis: A Review of Diagnostics Test for M. bovis Infection in Cattle", 2011, Agri-Food and Biosciences Institute, Belfast, Northern Ireland, retrieved from http:// www.daera-ni.gov.uk/sites/default/files/publications/dard/ afbi-literature-review-tb-review-diagnostic-tests-cattle.dpf.

7. J. S. Martinez, W. K. Grace, K. M. Grace, N. Hartman, and B. I. Swanson, J. Mater. Chem., 2005, 15, 4639.

8. H. Mukundan, J. Kubicek-Sutherland, A. Holt, J. E. Shively, J. S. Martinez, K. M. Grace, W. K. Grace, and B. I. Swanson, Sens. Actuators, B, 2009, 138, 453.

9. H. Mukundan, S. Kumar, D. N. Price, S. M. Ray, Y. J. Lee, S. Min, S. Eum, J. Kubicek-Sutherland, J. M. Resnick, W. K. Grace, A. S. Anderson, S. H. Hwang, S. N. Cho, L. E. Via, C. E. Barry 3rd, R. M. Sakamuri, and B. I. Swanson, Tuberculosis, 2012, 92, 407.

10. L. R. Stromberg, N. W. Hengartner, K. L. Swingle, R. A. Moxley, S. W. Graves, G. A. Montano, and H. Mukundan, PLOS ONE, 2016, e0156295.

11. R. R. Kale, H. Mukundan, D. N. Price, J. F. Harris, D. M. Lweallen, B. I. Swanson, J. G. Schmidt, and S. S. Iyer, J. Am. Chem. Soc., 2008, 130, 8169.

12. H. Mukundan, A. S. Anderson, W. K. Grace, K. M. Grace, N. Hartman, J. S. Martinez, and B. I. Swanson, Sensors, 2009, 9, 5783 .

13. H. Mukundan, H. Xie, A. S. Anderson, W. K. Grace, J. E. Shively, and B. I. Swanson, Bioconjug. Chem., 2009, 20, 222.

14. R. M. Sakamuri, D. N. Price, M. Lee, S. N. Cho, C. E. Barry 3rd, L. E. Via, B. I. Swanson, and H. Mukundan, Tuberculosis, 2013, 93, 301.

15. A. S. Anderson, A. M. Dattelbaum, G. A. Montano, D. N. Price, J. G. Schmidt, J. S. Martinez, W. K. Grace, K. M. grace, and B. I. Swanson, Langmuir, 2008, 24, 2240.

16. R. M. Sakamuri, P. Capek, T. J. Dickerson, C. E. Barry 3rd, and H. Mukundan, J. Microbiol. Meth., 2014, 103, 112.

17. D. Chatterjee and K. H. Khoo, Glycobiology, 1998, 8, 113.

18. T. Ito, A. Hasegawa, H. Hosokawa, M. Yamashita, S. Motohashi, T. Naka, Y. Okamoto, Y. Fujita, Y. Ishii, M. Taniguchi, I. Yano, and T. Nakayama, Int. Immunol., 2008, 20,849 .

19. G. V. Kanaujia, M. A. Garcia, D. M. Bouley, R. Peters, and M. M. Gennaro, Comparative Med., 2003, 53, 602.

20. X. Wang, P. F. Barnes, K. M. Dobos-Elder, J. C. Townsend, Y. T. Chung, H. Shams, S. E. Weiss, and B. Samten, $J$. Immunol., 2009, 182, 3668.

21. B. Hamasur, J. Bruchfeld, M. Haile, A. Pawlowski, B. Bjorvtan, G. Kallenius, and S. B. Svenson, J. Microbiol. Meth., 2001, 45, 41.

22. S. D. Lawn, BMC Infect. Dis., 2012, 12, 103.

23. B. Hamasur, J. Bruchfeld, P. van Helden, G. Kallenius, and S. B. Svenson, PLOS ONE, 2015, 10, e0123457.

24. J. Minion, E. Leung, E. Talbot, K. Dheda, M. Pai, and D. Menzies, Eur. Respir. J., 2011, 38. 